\title{
Field theory in normal conical coordinates
}

\author{
Dmitry Nesnov ${ }^{1, *}$ \\ ${ }^{1}$ Samara State Technical University, 244, Molodogvardeyskaya st., Samara, 443100, Russia
}

\begin{abstract}
In the scientific literature, the field theory is most fully covered in the cylindrical and spherical coordinate systems. This is explained by the fact that the mathematical apparatus of these systems is most well studied. When the source of field has a more complex structure than a point or a straight line, there is a need for new approaches to their study. The goal of this research is to adapt the field theory related to curvilinear coordinates in order to represent it in the normal conical coordinates. In addition, an important part of the research is the development of a geometrical modeling apparatus for scalar and vector field level surfaces using computer graphics. The paper shows the dependences of normal conical coordinates on rectangular Cartesian coordinates, Lame coefficients. The differential characteristics of the scalar and vector fields in normal conical coordinates are obtained: Laplacian of scalar and vector fields, divergence, rotation of the vector field. The example case shows the features of the application of the mathematical apparatus of geometrical field modeling in normal conical coordinates. For the first time, expressions for the characteristics of the scalar and vector fields in normal conical coordinates are obtained. Methods for geometrical modeling of fields using computer graphics have been developed to provide illustration in their study.
\end{abstract}

\section{Introduction}

Many different processes and phenomena are modeled using the mathematical apparatus of the field theory. The elements of the field theory are most fully represented in the vector presentation in rectangular Cartesian coordinates [1-2].

When the field sources have different geometrical shapes, it is convenient to describe them using special coordinate systems. For example, if the field source is concentrated at a point, its differential characteristics are conveniently described in the spherical coordinates. The obtained level surfaces will be spheres with the center in the field source (concentric spheres). If the source of the field is a straight line, then the differential characteristics will be described in the cylindrical coordinates. In this case, the level surfaces will be coaxial cylinders.

The field theory with point and linear sources is most fully investigated and described in the scientific literature [3]. This is because the describing mathematical apparatus is based on the use of spherical and cylindrical coordinate systems.

\footnotetext{
*Corresponding author: soft73@mail.ru
} 
When describing fields of complex structure, when the source has a shape different from a point or a straight line, mathematical complications arise. They require new research methods in studying the processes occurring in the environment of such fields $[4,5]$.

One of the simplest surfaces are a torus and a cone. The field theory in toroidal coordinates was previously described by the author in [6]. Differential characteristics of scalar and vector fields in normal toroidal coordinates were given. In this paper, the field theory in conical coordinates will be considered. When studying the level surfaces of thermal fields with a source in the form of external conical surfaces, it turned out that these surfaces do not coincide with families of equidistant cones. They are also not coordinate in any of the well-known coordinate systems. This is precisely the reason that significantly complicates the mathematical apparatus for describing fields and determining their characteristics.

The goal of this research is to introduce new coordinate systems for determining the characteristics of scalar and vector fields. When solving this problem, it is mandatory to preserve the condition of coincidence of coordinate families of surfaces with field level surfaces or with limiting surfaces of the process.

\section{Methods}

Functions that enter conical coordinates:

$$
\begin{gathered}
x=(u \cdot \sin \alpha+v \cdot \cos \alpha) \cos t ; y=(u \cdot \sin \alpha+v \cdot \cos \alpha) \sin t ; \\
z=u \cdot \cos \alpha-v \cdot \sin \alpha .
\end{gathered}
$$

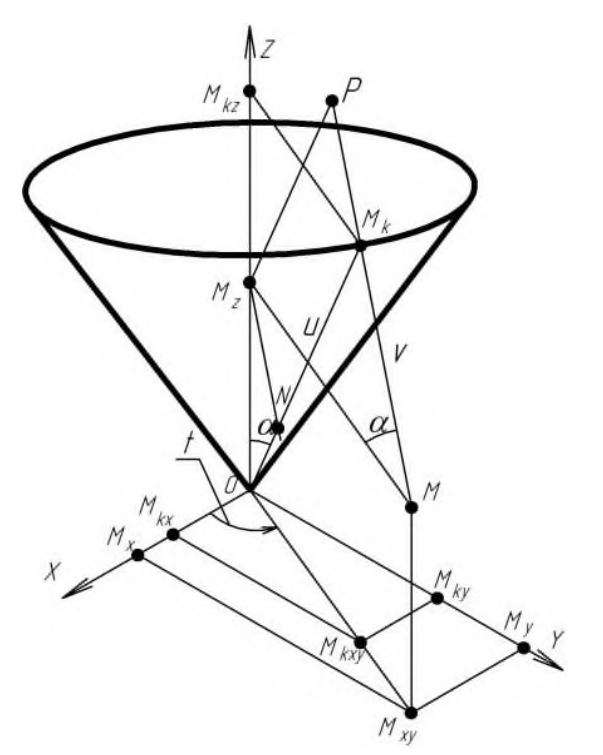

Fig. 1. Shows the cone-determinant.

Dependence functions of the normal conical coordinates $t, u, v$ on rectangular Cartesian coordinates. Since $t$ is a parameter of the half-plane, not the plane, to ensure uniqueness and to prevent division by zero in computer calculations, we determine the coordinate $t$ on the interval $0 \leq t \leq 2 \pi$ and add the above expressions for $u$ and $v$ 


$$
\begin{aligned}
& t=\frac{\pi}{2}, \text { if } x=0, y>0 ; \\
& t=\frac{3 \pi}{2}, \text { if } x=0, y<0 ; \\
& t=\operatorname{arctg} \frac{y}{x}, \text { if } x>0, y>0 ; \\
& t=\operatorname{arctg} \frac{y}{x}+\pi, \text { if } x<0 ; \\
& t=\operatorname{arctg} \frac{y}{x}+2 \pi, \text { if } x>0, y<0 ; \\
& u=\sqrt{x^{2}+y^{2}} \sin \alpha+z \cdot \cos \alpha ; \\
& v=\sqrt{x^{2}+y^{2}} \cos \alpha-z \cdot \sin \alpha .
\end{aligned}
$$

The condition for the possibility of the unique solution of equations (1) for $t, u, v$ and equations (2) for $x, y, z$

$$
\frac{D(x, y, z)}{D(t, u, v)}=\left|\begin{array}{lll}
\frac{\partial x}{\partial t} & \frac{\partial y}{\partial t} & \frac{\partial z}{\partial t} \\
\frac{\partial x}{\partial u} & \frac{\partial y}{\partial u} & \frac{\partial z}{\partial u} \\
\frac{\partial x}{\partial v} & \frac{\partial y}{\partial v} & \frac{\partial z}{\partial v}
\end{array}\right|=u \sin \alpha+v \sin \alpha \neq 0
$$

where partial derivatives are used for functions (1):

$$
\begin{array}{lll}
\frac{\partial x}{\partial t}=-(u \cdot \sin \alpha+v \cdot \cos \alpha) \sin t, & \frac{\partial y}{\partial t}=(u \cdot \sin \alpha+v \cdot \cos \alpha) \cos t, & \frac{\partial z}{\partial t}=0, \\
\frac{\partial x}{\partial u}=\sin \alpha \cdot \cos t, & \frac{\partial y}{\partial u}=\sin \alpha \cdot \sin t, & \frac{\partial z}{\partial u}=\cos \alpha \\
\frac{\partial x}{\partial v}=\cos \alpha \cdot \cos t, & \frac{\partial y}{\partial v}=\cos \alpha \cdot \sin t, & \frac{\partial z}{\partial v}=-\sin \alpha .
\end{array}
$$

Those points at which the expression of the Jacobian (3) is equal to zero,

$$
u \cdot \sin \alpha+v \cdot \cos \alpha=0
$$

make up a region of space whose coordination by normal conical coordinates is impossible. We define $v$ from (5)

$$
v=-u \operatorname{tg} \alpha
$$

The equation (6) corresponds to the axis of the cone, which coincides with the axis $O z$.

Another paper will consider the definition of the existence region of the introduced system of normal conical coordinates (the region of the correct coordination of space by normal conical coordinates).

Differential-geometrical characteristics of fields in normal conical coordinates

We find the expressions of partial derivatives of functions 


$$
\begin{aligned}
\frac{\partial x}{\partial t} & =-(u \sin \alpha+v \cos \alpha) \sin \left[t+\frac{(1-\operatorname{sign}(\mathrm{utg} \alpha+\mathrm{v})) \pi}{2}\right] \\
\frac{\partial y}{\partial t} & =(u \sin \alpha+v \cos \alpha) \cos \left[t+\frac{(1-\operatorname{sign}(\mathrm{utg} \alpha+\mathrm{v})) \pi}{2}\right] \\
\frac{\partial z}{\partial t} & =0 \\
\frac{\partial x}{\partial u} & =\sin \alpha \cos \left[t+\frac{(1-\operatorname{sign}(\mathrm{u} \operatorname{tg} \alpha+\mathrm{v})) \pi}{2}\right] \\
\frac{\partial y}{\partial u}= & \sin \alpha \sin \left[t+\frac{(1-\operatorname{sign}(\mathrm{u} \operatorname{tg} \alpha+\mathrm{v})) \pi}{2}\right] ; \\
\frac{\partial z}{\partial u}=\cos \alpha ; & \frac{\partial x}{\partial v}=\cos \alpha \cos \left[t+\frac{(1-\operatorname{sign}(\mathrm{utg} \alpha+\mathrm{v})) \pi}{2}\right] \\
\frac{\partial y}{\partial v} & =\cos \alpha \sin \left[t+\frac{(1-\operatorname{sign}(\mathrm{utg} \alpha+\mathrm{v})) \pi}{2}\right] \\
& \frac{\partial z}{\partial v}=-\sin \alpha .
\end{aligned}
$$

Lame coefficients for normal conical coordinates are determined by formulas (7):

$$
H_{t}=u \sin \alpha+v \cos \alpha, H_{u}=1, H_{v}=1 .
$$

Elementary moves:

- when changing only $t d S_{t}=(u \sin \alpha+v \cos \alpha) d t$;

- when changing only $u d S_{u}=d u$;

- when changing only $v d S_{v}=d v$.

Linear element:

$$
d S^{2}=(u \sin \alpha+v \cos \alpha)^{2} d t^{2}+d u^{2}+d v^{2} .
$$

The element of volume:

$$
d \tau=(u \cdot \sin \alpha+v \cdot \cos \alpha) d t d u d v .
$$

Calculations showed that the system of normal conical coordinates refers to triorthogonal systems. Since the coefficients $H_{t}, H_{u}, H_{v}$ satisfy six second-order partial differential equations, all terms of which are zero. That is, in such a system, each pair of coordinate surfaces intersects each other along the lines of curvature.

$$
\begin{gathered}
\frac{\partial}{\partial t}\left(\frac{1}{H_{t}} \frac{\partial H_{u}}{\partial t}\right)+\frac{\partial}{\partial u}\left(\frac{1}{H_{u}} \frac{\partial H_{t}}{\partial u}\right)+\frac{1}{H_{v}^{2}} \frac{\partial H_{t}}{\partial v} \frac{\partial H_{u}}{\partial v}=0 \\
\frac{\partial}{\partial u}\left(\frac{1}{H_{u}} \frac{\partial H_{v}}{\partial u}\right)+\frac{\partial}{\partial v}\left(\frac{1}{H_{v}} \frac{\partial H_{u}}{\partial v}\right)+\frac{1}{H_{t}^{2}} \frac{\partial H_{u}}{\partial t} \frac{\partial H_{v}}{\partial t}=0 \\
\frac{\partial}{\partial v}\left(\frac{1}{H_{v}} \frac{\partial H_{t}}{\partial v}\right)+\frac{\partial}{\partial t}\left(\frac{1}{H_{t}} \frac{\partial H_{v}}{\partial t}\right)+\frac{1}{H_{u}^{2}} \frac{\partial H_{v}}{\partial u} \frac{\partial H_{t}}{\partial u}=0
\end{gathered}
$$




$$
\begin{aligned}
& \frac{\partial^{2} H_{t}}{\partial u \partial v}-\frac{1}{H_{u}} \frac{\partial H_{u}}{\partial v} \frac{\partial H_{t}}{\partial u}-\frac{1}{H_{v}} \frac{\partial H_{v}}{\partial u} \frac{\partial H_{t}}{\partial v}=0 \\
& \frac{\partial^{2} H_{u}}{\partial v \partial t}-\frac{1}{H_{v}} \frac{\partial H_{v}}{\partial t} \frac{\partial H_{u}}{\partial v}-\frac{1}{H_{t}} \frac{\partial H_{t}}{\partial v} \frac{\partial H_{u}}{\partial t}=0 \\
& \frac{\partial^{2} H_{v}}{\partial t \partial u}-\frac{1}{H_{t}} \frac{\partial H_{t}}{\partial u} \frac{\partial H_{v}}{\partial t}-\frac{1}{H_{u}} \frac{\partial H_{u}}{\partial t} \frac{\partial H_{v}}{\partial u}=0
\end{aligned}
$$

Vector gradient of scalar field at points $t, u, v$, related to a moving local rapper

$$
G=\operatorname{grad} F=\frac{1}{(u \cdot \sin \alpha+v \cdot \cos \alpha)} \frac{\partial F}{\partial t} e_{t}+\frac{\partial F}{\partial u} e_{u}+\frac{\partial F}{\partial v} e_{v}
$$

The derivative of the scalar field in the direction $\bar{l}_{0}$

$$
\bar{l}_{0}=\cos \bar{\alpha} e_{t}+\cos \bar{\beta} e_{u}+\cos \bar{\gamma} e_{v}
$$

where $-\cos \bar{\alpha}, \cos \bar{\beta}, \cos \bar{\gamma}$ director cones of the unitary vector $\bar{l}_{0}$ with respect to $e_{t}, e_{u}, e_{v}$

$$
\frac{d F}{d l}=\frac{G_{t}}{|\bar{G}|} \cos \bar{\alpha}+\frac{G_{u}}{\mid \overline{|G|}} \cos \bar{\beta}+\frac{G_{v}}{|\bar{G}|} \cos \bar{\gamma} .
$$

where $G_{t}=\frac{1}{u \sin \alpha+v \cos \alpha} \frac{\partial F}{\partial t}, G_{u}=\frac{\partial F}{\partial u}, G_{v}=\frac{\partial F}{\partial v}, \overline{|G|}=\sqrt{G_{t}^{2}+G_{u}^{2}+G_{v}^{2}}$.

Laplacian of a scalar field with the account of the expressions $H_{t}, H_{u}, H_{v}(2.15)$

$$
\begin{aligned}
& \Delta F=\frac{1}{u \cdot \sin \alpha+v \cdot \cos \alpha}\left[\frac{\partial^{2} F}{\partial t^{2}} \frac{1}{u \cdot \sin \alpha+v \cdot \cos \alpha}+\right. \\
& \left.+\frac{\partial^{2} F}{\partial u^{2}}(u \cdot \sin \alpha+v \cdot \cos \alpha)+\frac{\partial F}{\partial u} \sin \alpha+\frac{\partial^{2} F}{\partial v^{2}} \cos \alpha\right]
\end{aligned}
$$

Vector flux $\bar{a}$ across the surface $S$ is determined by the formula:

$$
N=\iint_{S}\left[a_{t}(t, u, v) \cos \left(\bar{n}_{t} \wedge e_{t}\right)+a_{u}(t, u, v) \cos \left(\bar{n}_{u} \wedge e_{u}\right)+a_{v}(t, u, v) \cos \left(\bar{n}_{v} \wedge e_{v}\right)\right] d S
$$

where $\bar{n}$ - normal vector to the elementary area $d S$.

Definition of the formula is possible only after the presentation of the field and surface.

The divergence of the vector field is calculated taking into account the expressions of the Lame coefficients (8)

$$
\begin{gathered}
\operatorname{div} \bar{a}=\frac{1}{u \cdot \sin \alpha+v \cdot \cos \alpha}\left[\frac{\partial a_{t}}{\partial t}+(u \cdot \sin \alpha+v \cdot \cos \alpha) \frac{\partial a_{u}}{\partial u}+a_{u} \sin \alpha+\right. \\
\left.+(u \cdot \sin \alpha+v \cdot \cos \alpha) \frac{\partial a_{v}}{\partial v}+a_{v} \cos \alpha\right]
\end{gathered}
$$

After simplifications we get 


$$
\operatorname{div} \bar{a}=\frac{\frac{\partial a_{t}}{\partial t}+a_{u} \sin \alpha+a_{v} \cos \alpha}{u \cdot \sin \alpha+v \cdot \cos \alpha}+\frac{\partial a_{u}}{\partial u}+\frac{\partial a_{v}}{\partial v}
$$

As we see the divergence of the vector field is a scalar.

Laplacian of the vector field

$$
\begin{aligned}
\Delta \bar{a}= & {\left[\frac{1}{(u \cdot \sin \alpha+v \cdot \cos \alpha)^{2}} \frac{\partial^{2} a_{t}}{\partial t^{2}}+\frac{\partial^{2} a_{t}}{\partial u^{2}}+\frac{\partial^{2} a_{t}}{\partial v^{2}}+\frac{\sin \alpha}{u \cdot \sin \alpha+v \cdot \cos \alpha} \frac{\partial a_{t}}{\partial u}+\right.} \\
& \left.+\frac{\cos \alpha}{u \cdot \sin \alpha+v \cdot \cos \alpha} \frac{\partial a_{t}}{\partial v}\right] e_{t}+\left[\frac{1}{(u \cdot \sin \alpha+v \cdot \cos \alpha)^{2}} \frac{\partial^{2} a_{u}}{\partial t^{2}}+\frac{\partial^{2} a_{u}}{\partial u^{2}}+\frac{\partial^{2} a_{u}}{\partial v^{2}}+\right. \\
& \left.+\frac{\sin \alpha}{u \cdot \sin \alpha+v \cdot \cos \alpha} \frac{\partial a_{u}}{\partial u}+\frac{\cos \alpha}{u \cdot \sin \alpha+v \cdot \cos \alpha} \frac{\partial a_{u}}{\partial v}\right] e_{u}+ \\
& +\left[\frac{1}{(u \cdot \sin \alpha+v \cdot \cos \alpha)^{2}} \frac{\partial^{2} a_{v}}{\partial t^{2}}+\frac{\partial^{2} a_{v}}{\partial u^{2}}+\frac{\partial^{2} a_{v}}{\partial v^{2}}+\frac{\sin \alpha}{u \cdot \sin \alpha+v \cdot \cos \alpha} \frac{\partial a_{v}}{\partial u}+\right. \\
& \left.+\frac{\cos \alpha}{u \cdot \sin \alpha+v \cdot \cos \alpha} \frac{\partial a_{v}}{\partial v}\right] e_{v} .
\end{aligned}
$$

The rotation (the curl) of the vector field is determined taking into account the expressions $H_{t}, H_{u}, H_{v}(8)$

$$
\begin{aligned}
W_{t} & =\frac{\partial a_{v}}{\partial u}-\frac{\partial a_{u}}{\partial v} ; \\
W_{u} & =\frac{\partial a_{t}}{\partial v}+\frac{\cos \alpha}{u \cdot \sin \alpha+v \cdot \cos \alpha} a_{v}-\frac{1}{u \cdot \sin \alpha+v \cdot \cos \alpha} \frac{\partial a_{v}}{\partial t} \\
W_{v} & =\frac{1}{u \cdot \sin \alpha+v \cdot \cos \alpha} \frac{\partial a_{u}}{\partial t}-\frac{\partial a_{t}}{\partial u}-\frac{\sin \alpha}{u \cdot \sin \alpha+v \cdot \cos \alpha} a_{t} . \\
\operatorname{rot} \bar{a} & =\left(\frac{\partial a_{v}}{\partial u}-\frac{\partial a_{u}}{\partial v}\right) e_{t}+\left[\frac{\partial a_{t}}{\partial v}+\frac{1}{u \cdot \sin \alpha+v \cdot \cos \alpha}\left(a_{v} \cos \alpha-\frac{\partial a_{v}}{\partial t}\right)\right] e_{u}+ \\
& +\left[\frac{1}{u \cdot \sin \alpha+v \cdot \cos \alpha}\left(\frac{\partial a_{u}}{\partial t}-a_{t} \sin \alpha\right)-\frac{\partial a_{t}}{\partial u}\right] e_{v}
\end{aligned}
$$

The above expressions of the differential-geometrical characteristics of the scalar and vector fields, presented in normal conical coordinates, make it possible to design a geometrical model of the field and involve visual computer graphics in its study.

Let us show in a specific context the features of the application of the mathematical apparatus of the geometrical field modeling in normal conical coordinates.

Task. Calculate the derivative of the scalar field

$$
F=-0,25 \sin u \cos 6 t-v
$$

in the line of

$$
\bar{l}^{0}=3 e_{t}+4,8 e_{u}-2 e_{v}
$$

at point $M(t=0,4, u=10 v=-5)$. The system cone-determiner parameter $\alpha=0.52$. 
To show scalar field level surfaces (20)

$$
v=-0,25 \sin u \cos 6 t+i \Delta C
$$

with $i=0,1,2, \Delta C=0,4,0 \leq t \leq 2 \pi, 0,5 \leq u \leq 1,57$.

Solution. We find the direction cosines of the vector $\bar{l}_{0}$ in the basis $e_{t}, e_{u}, e_{v}$

$$
\begin{aligned}
& \cos \bar{\alpha}=\frac{3}{\sqrt{3^{2}+4,8^{2}+2^{2}}}=0,5 ; \cos \bar{\beta}=\frac{4,8}{\sqrt{3^{2}+4,8^{2}+2^{2}}}=0,8 \\
& \cos \bar{\gamma}=-\frac{2}{\sqrt{3^{2}+4,8^{2}+2^{2}}}=-0,3317 .
\end{aligned}
$$

We fine the coordinates $\mathrm{Gt}, \mathrm{Gu}, \mathrm{Gv}$ of the scalar field gradient vectors (20) according to formulas (12)

$$
G_{t}=-\frac{1}{u \sin \alpha+v \cos \alpha} 6(-0,25) \sin u \sin 6 t, G_{u}=-0,25 \cos u \cos 6 t, G_{v}=-1 .
$$

We substitute the values of the coordinates of the point $M$ and the parameter $\alpha$ of the cone-determinant. We get

$$
\begin{aligned}
& G_{t}=\frac{-6(-0,25) \sin (10) \sin (6 \cdot 0,4)}{10 \sin (0,52)-5 \cos (0,52)}=-0,8763 \\
& G_{u}=(-0,25) \cos (10) \cos (6 \cdot 0,4)=-0,1546 \\
& G_{v}=-1
\end{aligned}
$$

Gradient vector module

$$
G=\sqrt{(-0,8763)^{2}+(-0,1546)^{2}+1^{2}}=\sqrt{0,7679+0,0239+1}=1,3386 .
$$

We calculate the value of the derivative of the scalar field (20) in the direction (21) at the point $M$, using the formula (14) and the value of the direction cosines

$$
\frac{d F}{d l}=-\frac{0,8763}{1,3386} \cdot 0,5-\frac{0,1546}{1,3386} \cdot 0,8-\frac{1}{1.3386}(-0.3317)=-0.1653 .
$$

We build level surfaces according to internal equations (22).

Figure 2 shows three level surfaces together; in Figure 3, 4, 5 these surfaces are shown separately. 


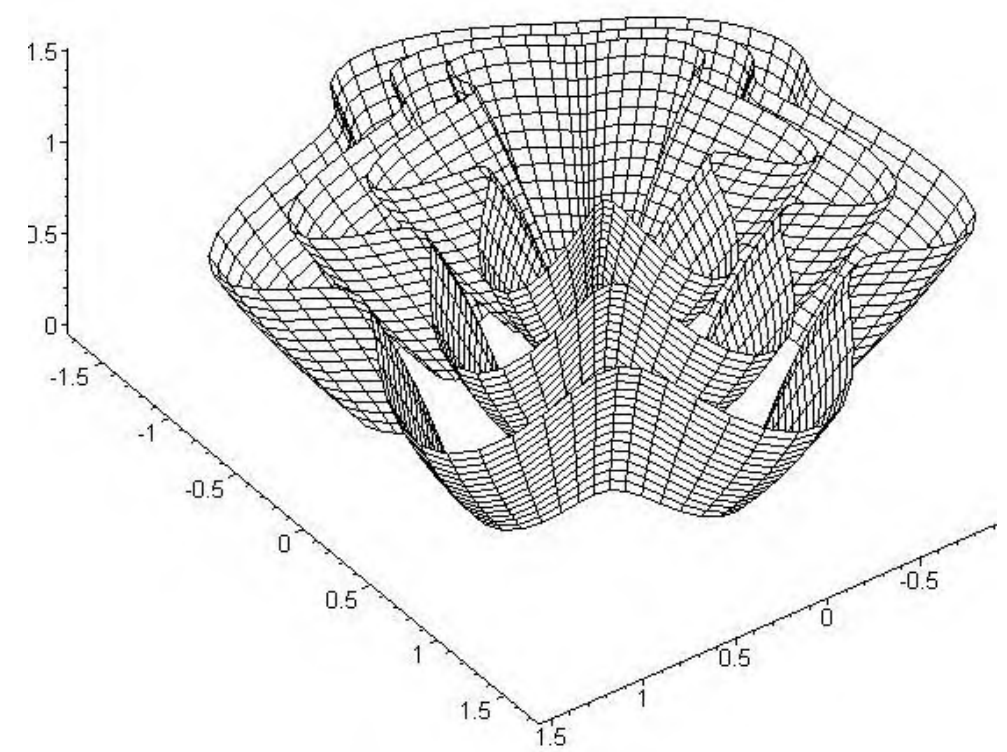

Fig. 2. Surfaces of the level $v=h \cdot \sin u \cdot \cos (n \cdot t)+i \Delta C$ of a scalar field

$F=h \cdot \sin u \cdot \cos (n \cdot t)-v$ with parameters $\Delta C=0,4 ; i=0,1,2 ; \alpha=0,52 ; h=-0,25 ; n=6 ; u=0,5 \ldots 1,57$; $t=0 . .2 \pi$

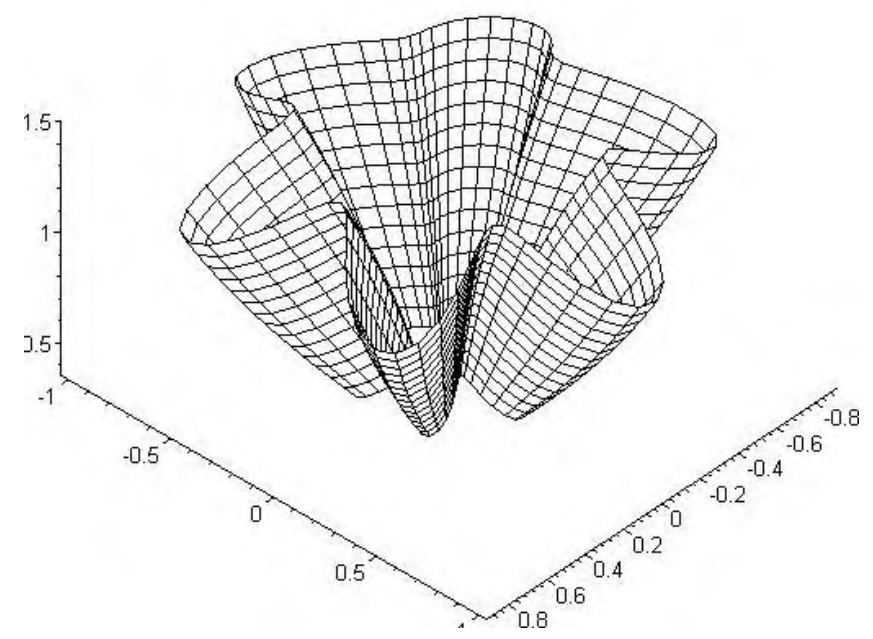

Fig. 3. Surfaces of the level $i=0$ 


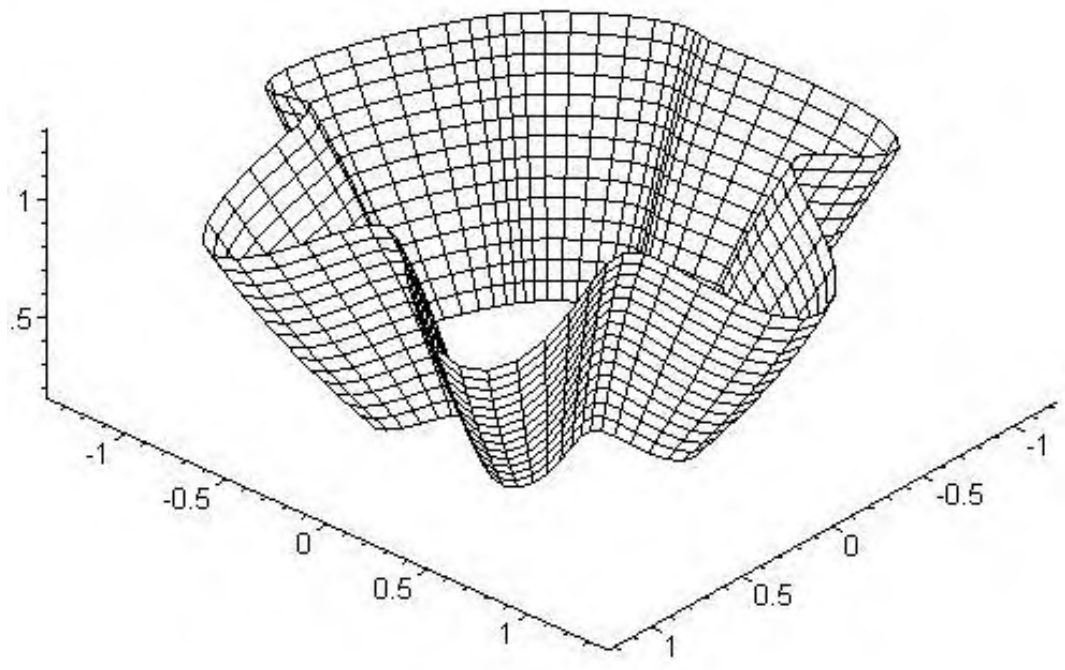

Fig. 4. Surfaces of the level $i=1$

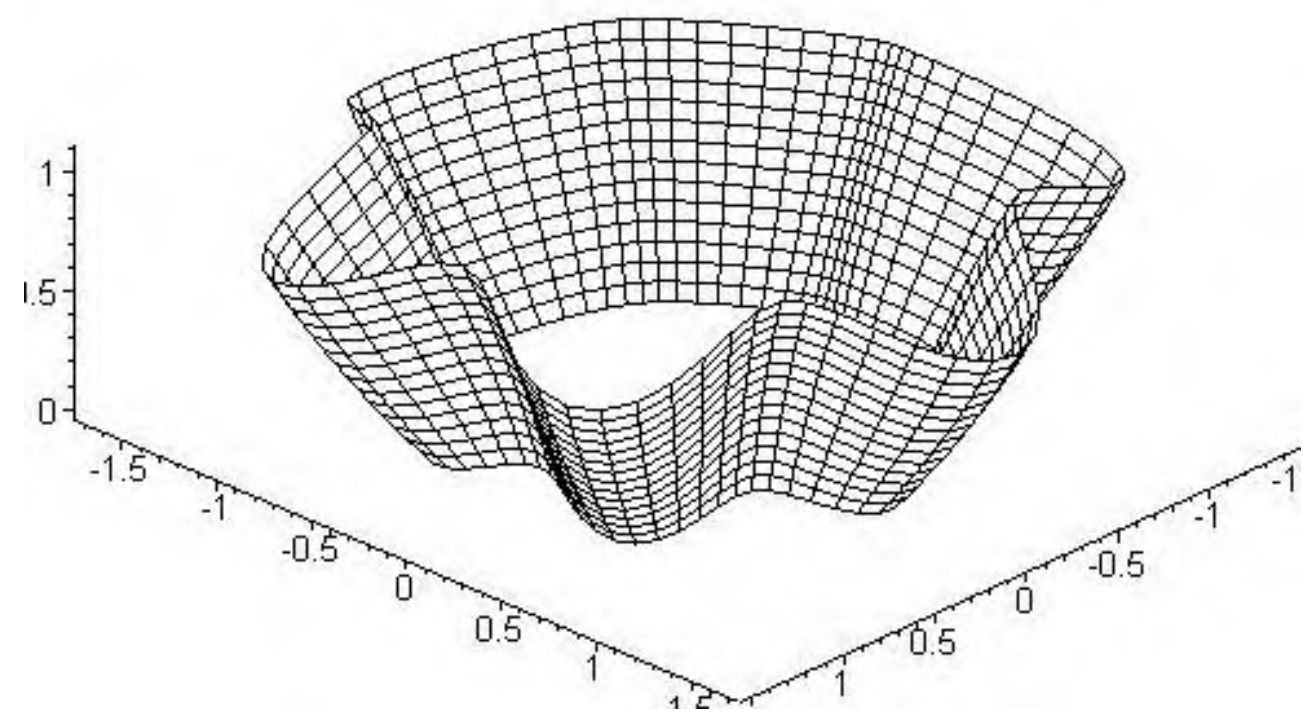

Fig. 5. Surfaces of the level $i=2$

\section{Results}

1. The paper gives the expressions of the differential characteristics of the scalar and vector fields in normal conical coordinates.

2. The methodology for calculating and constructing the level surfaces of the scalar fields represented in normal conical coordinates is shown.

\section{Discussion}

1. The obtained means of describing fields in normal conical coordinates allow visualizing, using computer graphics, the surfaces of a level with a complex structure. 
2. The example shows the practical application of the results to describe the level surfaces of the scalar fields.

3. The results of the theoretical research can be applied to solve various problems:

- obtaining the parameters of the scalar fields at a known point in space with the given parameters of the determinant cone and the subsequent visualization of level surfaces;

- calculation of vector field parameters (divergence, Laplacian, rotation) in normal conical coordinates;

- visualization of the field level surfaces in normal conical coordinates for various values of the parameter of the cone-determinant $\alpha$.

\section{Conclusion}

Using special coordinates to describe fields of complex structure greatly simplifies the calculation of field characteristics. A further development of this topic is the study of the region of existence of the introduced system of normal conical coordinates, which can also be called the region of proper coordination of normal conical coordinates.

\section{References}

1. L. D. Landau, E. M. Lifshitz, The classical theory of fields (Elsevier, New York, 2013)

2. P. Francesco, P. Mathieu, D. Senechal, Conformal field theory (Springer-Verlag, New York, 2012)

3. J. Quartieri, L. Sirignano, C. Guarnaccia, WSEAS Int. conf. (EMESEG'08), Heraklion, Greece (2008)

4. A. A. Tsinaeva, M. N. Nikitin, Procedia Eng. 150, 2340-2344 (2016), DOI: 10.1016/j.proeng.2016.07.321

5. M. N. Nikitin, J. of Physics: Conf. series 891, 12039 (2017), DOI: 10.1088/17426596/891/1/012039

6. D.V. Nesnov, Field theory in normal toroidal coordinates, MATEC Web of Conferences, Vol. 193, 003022 (2018) DOI :10.1051/matecconf/201819303022

7. F. Aggogeri, A. Avanzini, A. Borboni, S. Pandini, International Journal of Automation Technology 11(2), 311-321 (2017) DOI: 10.20965/ijat.2017.p0311

8. I. Ilin, V. Vasilenok, R. Marchenko, Use of renewable energy and tax burden on CO2 emissions in industrial enterprises, E3S Web of Conferences, 110, 0210 (2019) 